\title{
Efeitos agudos da aplicação endovenosa do cogumelo-do- sol (Agaricus blazei Murill) sobre a pressão arterial média e a freqüência cardíaca de ratos anestesiados
}

\author{
Glenan Singi $^{1 *}$, Dênis D. Damasceno ${ }^{1}$, Éverton D. D'Andréa ${ }^{1}$, Giovana M.B. Alexandre ${ }^{1}$, \\ Marianne B. Singi ${ }^{1}$, Lira C. Alves ${ }^{2}$, Tiessa I. Simões ${ }^{2}$ \\ ${ }^{1}$ Departamento de Ciências Biológicas, Universidade Federal de Alfenas - UNIFAL - Rua Gabriel \\ Monteiro da Silva, 714, Centro, 37130-000, Alfenas, MG, Brasil, \\ ${ }^{2}$ Departamento de Ciencias Exatas, Universidade Federal de Alfenas - UNIFAL, Rua Gabriel Monteiro da Silva, \\ 714, Centro, 37130-000, Alfenas, $M G$, Brasil
}

\begin{abstract}
RESUMO: Este trabalho objetivou verificar os efeitos agudos da aplicação endovenosa do extrato aquoso do Agaricus blazei Murill sobre a pressão arterial média (PAM) e a freqüência cardíaca (FC) de ratos anestesiados. Foram usados Rattus novergicus albinus, $\mathrm{n}=6$, anestesiados com tiopental sódico, traqueostomizados e canulados através da veia jugular e da artéria carótida. Foram injetadas as concentrações de $1,25 \mathrm{mg} / \mathrm{kg}, 2,50 \mathrm{mg} / \mathrm{kg}$ e $5,00 \mathrm{mg} / \mathrm{kg}$ do extrato aquoso em volume de $0,2 \mathrm{~mL}$. A PAM foi registrada com um sistema Biopac, modelo MP100, e a FC com um eletrocardiógrafo ECG-4 Funbec. Os resultados foram obtidos no controle e nos tempos 15, 30, 45, 60 e 120s após a aplicação dos extratos. Os valores foram expressos em média $\pm \mathrm{EPM}$ e analisados estatisticamente pelos testes " $\mathrm{t}$ " de Student-Newman-Keuls e Tukey $(\mathrm{p}<0,05)$. O extrato aquoso de $A$. blazei reduziu a PAM de maneira concentração dependente, sendo que a concentração de $1,25 \mathrm{mg} / \mathrm{kg}$ não provocou modificações significativas na PAM nem na FC; a de $2,50 \mathrm{mg} / \mathrm{kg}$ provocou diminuição da PAM aos $15 \mathrm{~s}(\mathrm{p}<0,01)$ e da FC aos $30 \mathrm{~s}(\mathrm{p}<0,001)$ e a de $5,00 \mathrm{mg} / \mathrm{kg}$ diminuiu a PAM aos $15 \mathrm{~s}(\mathrm{p}<0,001)$ e a $\mathrm{FC}$ aos 15 e $30 \mathrm{~s}(\mathrm{p}<0,001)$.
\end{abstract}

Unitermos: Agaricus blazei, cogumelo-do-sol, pressão arterial.

\begin{abstract}
Acute effects of the endovenous application of the royal mushroom (Agaricus blazei Murill) on mean arterial pressure and heart rate of anesthetized rats". The aim of this paper was to verify the acute effects of the endovenous application of the aqueous extract of Agaricus blazei Murill on mean arterial pressure and heart rate of the anesthetized rats. The injected concentrations were: $1.25 \mathrm{mg} / \mathrm{kg}, 2.50 \mathrm{mg} / \mathrm{kg}$ and $5.00 \mathrm{mg} / \mathrm{kg}$, in volume of $0.2 \mathrm{~mL}$. The rats were anesthetized with sodium thiopental and, after tracheotomy, both jugular vein and carotid artery were cannulated. The MAP was recorded with a Biopac System, model MP100. The HR was obtained with an electrocardiograph model ECG-4 (Funbec). The records were made in the control and 15,30,45, 60 and 120s after the application of the different concentrations of the extracts. The values were expressed by mean \pm SEM and by paired " $t$ "-Student-Newman-Keuls and Tukey tests $(\mathrm{p}<0.05)$. The aqueous extract of the $A$. blazei decreased the MAP of dependent manner. The concentration of $1.25 \mathrm{mg} / \mathrm{kg}$ did not provoke effects; $2.50 \mathrm{mg} / \mathrm{kg}$ provoked decrease of the PAM at $15 \mathrm{~s}(\mathrm{p}<0.01)$ and of the HR at $30 \mathrm{~s}(\mathrm{p}<0.001)$ and $5.00 \mathrm{mg} / \mathrm{kg}$ provoked decrease of the PAM at $15 \mathrm{~s}$ $(p<0.001)$ and of the HR at 15 and $30 \mathrm{~s}(\mathrm{p}<0.001)$.
\end{abstract}

Keywords: Agaricus blazei, royal mushroom, arterial pressure.

\section{INTRODUÇÃO}

O Agaricus blazei Murill é um cogumelo extensivamente produzido e consumido como medicamento e alimento popular devido ao seu valor medicinal, sendo usado como um estimulante do sistema imunológico e no tratamento de várias doenças, incluindo o câncer (Ribeiro; Salvadori, 2003; Verçosa Júnior et al., 2004). É utilizado também para doenças cardíacas, diabetes e artrites, dentre outras (Lee et al., 2000; Ferreira et al., 2003). No Brasil é empregado tradicionalmente como um alimento para a prevenção do câncer, da hiperlipidemia, da arteriosclerose e da hepatite crônica (Kymura et al., 2004). Não deve ser confudido com o cogumelo tibetano, um consórcio microbiano nem com outras associações simbióticas da mesma natureza, como Kumish e o cogumelo kombucha, presentes na medicina popular de países asiáticos (Cardoso et al., 2005).

O basidiocarpo do A. blazei apresenta $40-45 \%$ de proteínas, $38-45 \%$ de carboidratos, $6-8 \%$ de fibras, $5-$ $7 \%$ de minerais e $3-5 \%$ de gordura, valores baseados em relação à matéria seca, contendo ainda teores elevados 
de potássio e cálcio (Mizuno et al., 1990). Em trabalho realizado por Kimura et al. (2002), foi identificada a presença dos aminoácidos valina e prolina, bem como a do mediador químico ácido gama-aminobutírico (GABA).

A regulação da ativação e proliferação de células$\mathrm{T}$, a secreção de citocinas e a produção de óxido nítrico (NO) são alguns dos mecanismos de ação do $A$. blazei (Kuo et al., 2002). Em estudos realizados por Sorimachi et al. (2001), in vitro, foi mostrado que os componentes do A. blazei Murill que ativam macrófagos induzem a secreção de citocinas (IL-8 e TNF- $\alpha$ ) e NO.

Apesar de ter sido relatado o seu uso em doenças cardíacas (Lee et al., 2000), a literatura consultada não mostrou nenhum estudo da atuação do A. blazei sobre a pressão arterial.

A hipertensão arterial é uma doença altamente prevalente em nosso meio, atingindo cerca de 15 a $20 \%$ da população adulta com mais de 18 anos, chegando a alcançar índices de 50\% nos idosos (Mion Jr et al., 2001; Barbosa-Filho et al., 2006). Segundo Waib et al. (1992), tem crescido o interesse por medidas terapêuticas não farmacológicas que possam auxiliar no controle da hipertensão arterial.

O presente estudo objetivou avaliar o efeito agudo do A. blazei sobre a pressão arterial média (PAM) e a freqüência cardíaca (FC) de ratos anestesiados, criando a possibilidade de um futuro estudo de sua utilização crônica.

\section{MATERIAL E MÉTODOS}

Ratos machos, adultos (Rattus novergicus albinus) $(\mathrm{n}=6)$, pesando em média 400 gramas, com idade de 6 meses, provenientes do biotério da UNIFALMG, mantidos em jejum de 12 horas, foram utilizados como grupo experimental, de acordo com metodologia descrita por Carbajal et al. (1989), tendo sido os animais anestesiados com tiopental sódico $(30 \mathrm{mg} / \mathrm{kg}$, intraperitoneal) e, em seguida, traqueostomizados e realizada canulação da veia jugular direita para aplicação dos extratos e da artéria carótida esquerda para o registro da PAM. Após o procedimento cirúrgico, aguardava-se a estabilização das variáveis hemodinâmicas, o que ocorria em torno de 15 minutos.

O A. blazei, proveniente da Fazenda Cardosas, Boa Esperança-MG-Brasil, foi seco e triturado e, para a obtenção do seu extrato aquoso, foram dissolvidos 2 gramas em $150 \mathrm{~mL}$ de água destilada, sendo a solução colocada em banho-maria a $100{ }^{\circ} \mathrm{C}$ por 2 horas, sob refluxo. Depois de resfriada, foi centrifugada e acertado o seu $\mathrm{pH}$ para 7,4. Foram preparadas e administradas as concentrações de $1,25 \mathrm{mg} / \mathrm{kg}, 2,50 \mathrm{mg} / \mathrm{kg}$ e $5,00 \mathrm{mg} /$ $\mathrm{kg}$. O volume injetado foi de $0,2 \mathrm{~mL}$ em 5 segundos, seguindo-se a aplicação de $0,2 \mathrm{~mL}$ de solução fisiológica para lavagem de possíveis resíduos na cânula. Antes da aplicação da concentração inicial, era injetado $0,4 \mathrm{~mL}$ de solução fisiológica $(0,2 \mathrm{~mL}+0,2 \mathrm{~mL})$, simulando a aplicação do extrato, que servia como controle das condições hemodinâmicas dos animais.Quando estas condições eram instáveis, os animais eram descartados.

O registro da PAM foi obtido através de um transdutor de pressão modelo TSD 104A, acoplado a um sistema Biopac MP 100 (Santa Bárbara-Califórnia), o qual foi ligado a um computador, possibilitando o registro em $\mathrm{mmHg}$. A FC foi medida por meio de um eletrocardiógrafo ECG-4 (Funbec), posicionando-se os eletrodos nas derivações clássicas dos membros. Os resultados obtidos foram expressos como médias e erros padrões das médias e analisados estatisticamente pelos testes " $t$ " de Student-Newman-Keuls e Tukey, no controle (tempo 0) e nos tempos de 15, 30, 45, 60 e 120 segundos após a aplicação dos extratos, sendo considerados significativos valores para $\mathrm{p}<0,05$.

\section{RESULTADOS E DISCUSSÃO}

A aplicação da solução fisiológica, que serviu para verificar as condições hemodinâmicas dos animais, não provocou alterações na PAM nem na FC (Fig.1).

A aplicação endovenosa da concentração de $1,25 \mathrm{mg} / \mathrm{kg}$ do extrato aquoso do $A$. blazei não produziu nenhum efeito sobre a PAM (Tab.1, Fig.2).

A aplicação endovenosa da concentração de 2,50 $\mathrm{mg} / \mathrm{kg}$ do extrato aquoso do $A$. blazei provocou redução da PAM aos $15 \mathrm{~s}(\mathrm{p}<0,01)$ (Tab.1, Fig.3).

A aplicação endovenosa da concentração de 5,00 $\mathrm{mg} / \mathrm{kg}$ do extrato aquoso do $A$. blazei provocou redução da PAM aos 15s (p<0,001) (Tab.1, Fig.4).

Estudo comparativo entre as concentrações mostrou que $1,25 \mathrm{mg} / \mathrm{kg}$ foi diferente de $2,50 \mathrm{mg} / \mathrm{kg}$ $(\mathrm{p}<0,05)$ e de $5,00 \mathrm{mg} / \mathrm{kg}(\mathrm{p}<0,01)$ e que $2,50 \mathrm{mg} / \mathrm{kg}$ foi diferente de $5,00 \mathrm{mg} / \mathrm{kg}(\mathrm{p}<0,05)$, mostrando efeito concentração dependente.

A concentração de $1,25 \mathrm{mg} / \mathrm{kg}$ não provocou alteração da FC de ratos anestesiados (Tab.2). A concentração de $2,50 \mathrm{mg} / \mathrm{kg}$ provocou diminuição da FC aos 30s $(p<0,01)$ e aos 45s $(p<0,05)$ (Tab.2). A concentração de $5,00 \mathrm{mg} / \mathrm{kg}$ também provocou diminuição da $\mathrm{FC}$ aos 15 e 30s $(\mathrm{p}<0,01)$ e aos 45, 60 e $120 \mathrm{~s}(\mathrm{p}<0,05)($ Tab.2).

Apesar do presente trabalho não ter objetivado explicar o mecanismo envolvido com a diminuição da PAM pelo $A$. blazei, podem-se fazer algumas especulações a respeito. Possivelmente a queda da pressão arterial se deu por secreção de NO, que é um potente vasodilador e neurotransmissor (Mizuno et al., 2000).

A diminuição da PAM pode também ser atribuída ao GABA, que é encontrado no A. blazei (Kimura et al., 2002), quer por ação direta sobre os vasos sanguíneos, quer por bloqueio ganglionar, com conseqüente inibição da liberação de transmissores nos terminais nervosos simpáticos, ou ainda, pela somação destes dois efeitos.

De acordo com Mizuno et al.(1990), o $A$. blazei apresenta elevados teores de potássio e cálcio. O 
cálcio pode ativar canais de potássio (BK channel) da musculatura lisa dos vasos, provocando hiperpolarização e relaxamento (Sun et al., 1994). Tal efeito não pode ser descartado na explicação da diminuição da pressão arterial provocada pelo $A$. blazei.

Para se entender os mecanismos envolvidos com a queda da PAM provocada pelo $A$. blazei, experimentos futuros deverão ser realizados utilizando-se bloqueadores de indutores de vasodilatação. Talvez eles estejam também envolvidos com a diminuição da FC.

\section{CONCLUSÃO}

A administração aguda endovenosa do extrato aquoso do Agaricus blazei Murill provocou diminuição concentração dependente da pressão arterial média. A freqüência cardíaca também sofreu diminuição, mas não concentração dependente.

Tabela 1. Efeitos da aplicação do extrato aquoso do Agaricus blazei sobre pressão arterial média de ratos anestesiados. Valores expressos em média \pm EPM.

\begin{tabular}{lcccccc}
\hline Concentrações & Controle & $15 \mathrm{~s}$ & $30 \mathrm{~s}$ & $45 \mathrm{~s}$ & $60 \mathrm{~s}$ & $120 \mathrm{~s}$ \\
$1,25 \mathrm{mg} / \mathrm{kg}$ & $122 \pm 4$ & $114 \pm 3$ & $112 \pm 4$ & $111 \pm 4$ & $111 \pm 4$ & $116 \pm 2$ \\
$2,50 \mathrm{mg} / \mathrm{kg}$ & $128 \pm 2$ & $110 \pm 3^{*}$ & $120 \pm 3$ & $124 \pm 4$ & $125 \pm 4$ & $122 \pm 2$ \\
$5,00 \mathrm{mg} / \mathrm{kg}$ & $121 \pm 3$ & $90 \pm 5^{* *}$ & $108 \pm 3$ & $111 \pm 3$ & $110 \pm 3$ & $111 \pm 3$ \\
\hline
\end{tabular}

* significativo para $\mathrm{p}<0,01$ em relação ao controle.

** significativo para $\mathrm{p}<0,001$ em relação ao controle.

Tabela 2. Efeitos da aplicação do extrato aquoso do Agaricus blazei sobre a freqüência cardíaca de ratos anestesiados. Valores expressos em média \pm EPM.

\begin{tabular}{lcccccc}
\hline Concentrações & Controle & $15 \mathrm{~s}$ & $30 \mathrm{~s}$ & $45 \mathrm{~s}$ & $60 \mathrm{~s}$ & $120 \mathrm{~s}$ \\
$1,25 \mathrm{mg} / \mathrm{kg}$ & $375 \pm 3$ & $375 \pm 3$ & $375 \pm 3$ & $375 \pm 3$ & $375 \pm 3$ & $375 \pm 3$ \\
$2,50 \mathrm{mg} / \mathrm{kg}$ & $375 \pm 3$ & $338 \pm 22$ & $311 \pm 6^{* *}$ & $322 \pm 6^{*}$ & $331 \pm 6$ & $331 \pm 6$ \\
$5,00 \mathrm{mg} / \mathrm{kg}$ & $375 \pm 3$ & $311 \pm 6^{* *}$ & $311 \pm 6^{* *}$ & $320 \pm 11^{*}$ & $320 \pm 11^{*}$ & $320 \pm 11^{*}$ \\
\hline
\end{tabular}

* significativo para $\mathrm{p}<0,05$ em relação ao controle.

** significativo para $\mathrm{p}<0,01$ em relação ao controle.

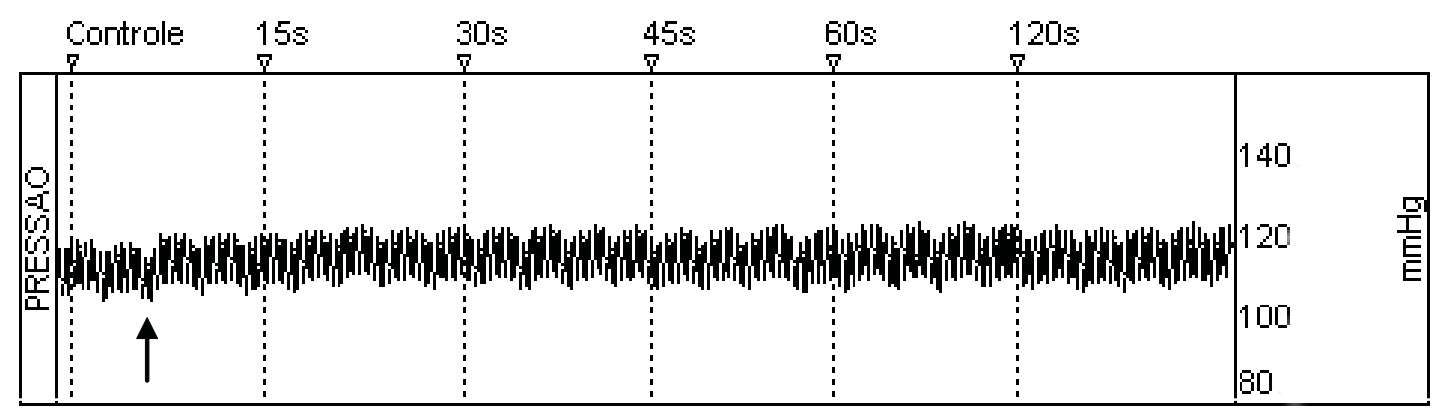

Figura 1. Efeitos da aplicação de solução fisiológica (seta) sobre a pressão arterial média de ratos anestesiados.

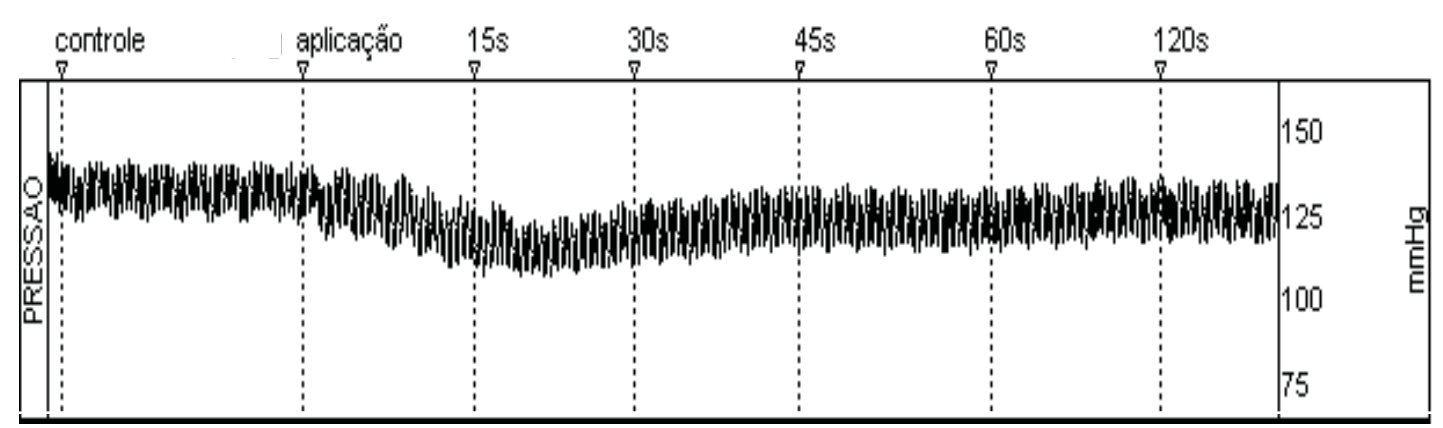

Figura 2. Efeitos da aplicação de $1,25 \mathrm{mg} / \mathrm{kg}$ do $A$. blazei sobre a pressão arterial média de ratos anestesiados. 


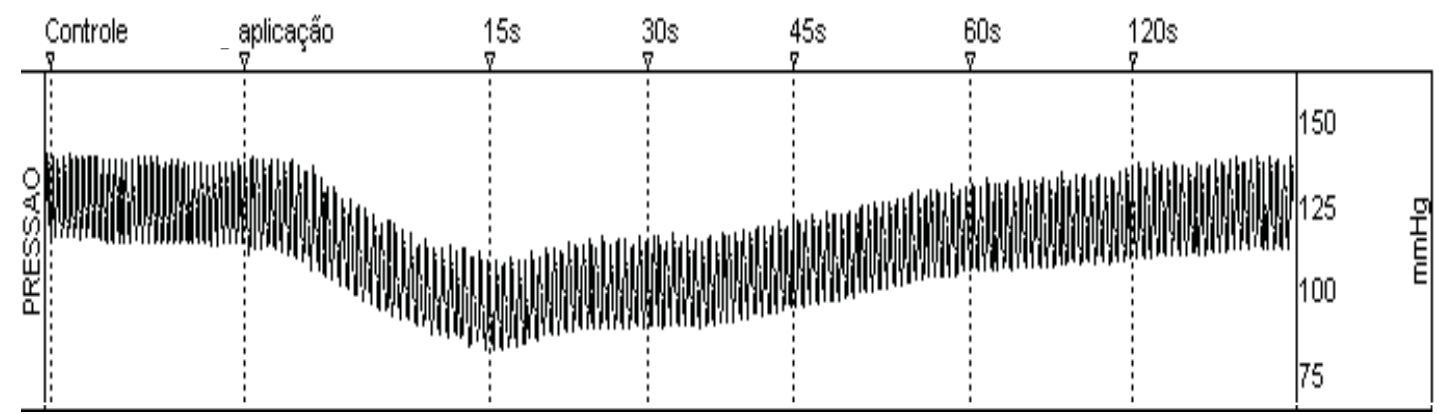

Figura 3. Efeitos da aplicação de $2,50 \mathrm{mg} / \mathrm{kg}$ do $A$. blazei sobre a pressão arterial média de ratos anestesiados.

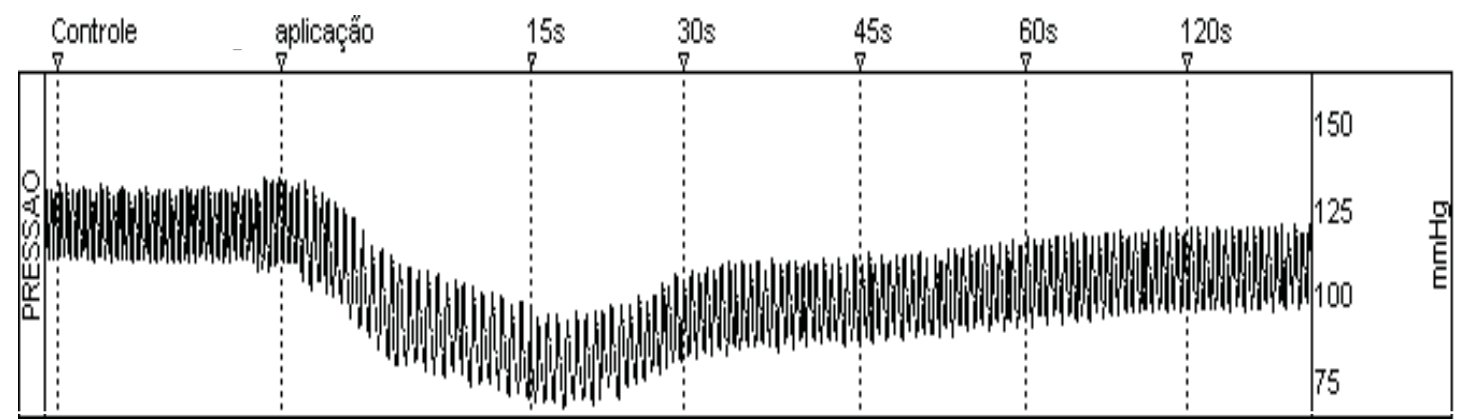

Figura 4. Efeitos da aplicação de $5,00 \mathrm{mg} / \mathrm{kg}$ do $A$. blazei sobre a pressão arterial média de ratos anestesiados.

\section{AGRADECIMENTOS}

Agradecemos ao Auxiliar-Técnico do Laboratório de Farmacologia e Fisiologia da UNIFALMG, Fernando Ponciano.

\section{REFERÊNCIAS}

Barbosa-Filho JM, Martins VKM, Rabelo LA, Moura MD, Silva MS, Cunha EVL, Souza MFV, Almeida RN, Medeiros IA 2006. Natural products inhibitors of the angiotensin converting enzyme (ACE). A review between 1980-2000. Rev Bras Farmacogn 16: 421446.

Carbajal D, Casaco A, Arruzababala L, Gonzalez R, Tolon T 1989. Pharmacologycal study of Cymbopogon citratus leaves. J Ethnopharmacol 25: 103-107.

Cardoso LGV, Schneedorf JM, Fiorini JE, Oliveira BR, Carvalho JCT 2005. Efeito da administração de cogumelo tibetano, um consórcio microbiano, sobre a peristalse intestinal em ratos. Rev Bras Farmacogn 15: 212-214.

Ferreira KM, Melo MM, Dantas-Barros AM 2003. Tratamento tópico de coelhos com Agaricus blazei Murril após envenenamento botrópico experimental. Rev Bras Farmacogn 13(Supl. 1): 77-80.

Kimura M, Hayakawa K, Sansawa H 2002. Involvement of -aminobutyric acid (GABA) B receptors in the hypotensive effect of systemically administered GABA in spontaneously hypertensive rats . Jpn $J$
Pharmacol 89: 388-394.

Kuo Y, Huang Y, Chen C, Lin Y, Chuang K, Tsai W 2002. Cell cycle progression and cytokine gene expression of human peripheral blood mononuclear cells modulated by Agaricus blazei. J Lab Clin Med 140: 176-187.

Kymura Y, Kido T, Takaku T, Sumiyoshi M, Baba K 2004. Isolation of an anti-angiogenic substance from Agaricus blazei Murill: Its antitumor and antimetastatic actions. Cancer Sci 95: 758-764.

Lee K, Wang H, Itokawa H, Morris-Natschke SL 2000. Current perspectives on Chinese medicines and dietary supplements in China, Japan and the United States. $J$ Food Drug Anal 8: 219-228.

Mion Jr D, Pierin AMS, Guimarães A 2001. Tratamento da hipertensão arterial. Respostas de médicos brasileiros a um inquérito. Rev Ass Med Bras 47: 249-254.

Mizuno T, Hagiwara T, Nakamura T, Ito H, Shimura K, Sumiya T, Asakura A 1990. Antitumor activity and some properties of water-soluble polysaccharides from "Himematsutake", the fruiting body of Agaricus blazei Murill. Agr Biol Chem 54: 2889-2896.

Mizuno M, Shiomi Y, Minato K, Kawakami S, Ashida H, Tsuchida H 2000. Fucogalactan isolated from Sarcodon aspratus elicits release of tumor necrosis factor-a and nitric oxide from murine macrophages. Immunopharmacol 46: 113-121.

Ribeiro LR, Salvadori DMF 2003. Dietary components may prevent mutation - related diseases in humans. Mutat Res-Rev Mutat 544: 195-201.

Sorimachi K, Akimoto K, Ikehara Y, Inafuku K, Okubo A, Yamazaki S 2001. Secretion of TNF- $\alpha$, IL-8 and nitric 
oxide by macrophages activated with Agaricus blazei Murill fractions in vitro. Cell Struct Funct 26: 103108.

Sun AD, Kim YB, Lee YH, Kang BS, Kang DH 1994. Fatty acids directly increase the activity of $\mathrm{Ca}^{2+}$ - activated $\mathrm{K}^{+}$channels in rabbit coronary smooth muscle cells. Yonsei Med J 35: 10-24.

Verçosa Júnior D, Melo MM, Dantas-Barros AM, Gomes AM, Silva Junior PG, Lago EP 2004. Quadro hematologico e peso do baço de camundongos com tumor de Ehrlich na forma sólida tratados com Agaricus blazei. Rev Bras Farmacogn 14(Supl. 1): 32-34.

Waib PH, Papini-Berto SJ, Habermann F, Burini RC 1992. Avaliação da ingestão dietética de cálcio em indivíduos adultos portadores de hipertensão arterial idiopática. Rev Saúde Pública 26: 27-33. 